\title{
Validación de la escala OCDUMA para analizar las concepciones, opiniones y percepciones del profesorado hacia las metodologías activas
}

\author{
Ana Rosa Arias-Gago \\ Universidad de León, España \\ mail: ana.ariasQunileon.es \\ ORCID: https://orcid.org/0000-0002-5889-3222 \\ Alejandro Rodríguez-García \\ Universidad de León, España \\ mail: arodrg01destudiantes.unileon.es \\ ORCID: https://orcid.org/0000-0002-7258-8857
}

\section{RESUMEN}

Las metodologías activas, en el actual sistema educativo, cada vez adquieren más relevancia como consecuencia del cambio de paradigma educativo motivado por los movimientos de renovación pedagógica impulsados en las enseñanzas universitarias y no universitarias. Este trabajo versa sobre el diseño y validación de un instrumento con el que determinar las opiniones, concepciones y percepciones de la población de profesorado no universitario español hacia estas metodologías activas. El instrumento se trata de una escala de observación conformada por 18 ítems, agrupados en 5 dimensiones, en las que la fiabilidad, bondad de ajuste y validez fueron analizadas en una muestra de 12490 docentes. Los resultados arrojaron unas propiedades psicométricas idóneas, que manifiestan consistencia y coherencia del modelo con los constructos teóricos de partida, lo que otorga una gran utilidad a este instrumento construido, validado y dirigido hacia los docentes no universitarios, ámbito en el que los instrumentos se caracterizan por el carácter exiguo.

Palabras clave: método de enseñanza; validación; fiabilidad; enseñanza no universitaria.

\section{Validation of the OCDUMA scale to analyze teachers' conceptions, opinions and perceptions towards ac- tive methodologies}

\begin{abstract}
Nowadays active methodologies are becoming increasingly relevant in the educative system as consequence of the educational paradigm shift motivated by the pedagogical renewal movements promoted from both university and non-university teaching. This work deals with the design and validation of an instrument that will determine the Spanish non-university teachers' population opinions, conceptions and perceptions towards these active methodologies. The instrument is an observation scale composed of 18 items grouped into 5 categories, whose reliability, validity and goodness fit were tested in a sample of 12490 teachers. The results show suitable psychometric properties, which demonstrate consistency and coherence of the model with the theoretical starting constructs, which gives great utility to this instrument, constructed, validated and directed towards non-university teachers, an area where the instruments are characterized by their scarce nature.
\end{abstract}

Keywords: teaching method; validation; reliability; non-university teaching. 


\section{Introducción}

La relevancia de la metodología en el ámbito educativo es un hecho que está fuera de toda duda. De este modo, como consecuencia de los movimientos de renovación pedagógica de la última década, se ha promovido un cambio de paradigma que afecta a todas las etapas educativas (Nurash, Kasevayuth \& Intarakamhang, 2020; Oliver, McConney \& Woods-McConney, 2019). Asimismo, De Miguel (2005), establece que a nivel educativo son ejemplos ilustrativos de este cambio de paradigma el proceso de convergencia de la Universidad al Espacio Europeo de Educación Superior y el currículo por competencias de la Educación no Universitaria.

Ambas iniciativas han traído consigo el cambio de un paradigma tradicional -que busca el aprendizaje conceptual del alumnado a través de sesiones magistrales en las que el docente es el protagonista- a un paradigma activo -en el que el docente actúa como regulador de los procesos de aprendizaje del alumnado, quien se erige como la figura central del aprendizaje- (De Miguel, 2005; Gil, Cordero \& Cristóbal 2018).

De este enfoque activo y/o moderno surgen las metodologías activas, las cuales son definidas como: "aquellos métodos, técnicas y estrategias que utiliza el docente para convertir el proceso de enseñanza-aprendizaje en actividades que fomenten la participación activa del estudiante y su protagonismo" (Labrador-Piquer \& Andreu-Andrés, 2008, p. 6). Estas metodologías activas disponen de un marcado carácter constructivista y fomentan el aprendizaje competencial extrapolando los conocimientos adquiridos a situaciones de la vida cotidiana (Fernández-de-Álava \& Quesada-Pallarés, 2017; OCDE, 2019; Rodríguez-García \& Arias-Gago, 2019).

En la última década, como consecuencia del desarrollo de las tecnologías de la información y de la comunicación (TIC) y del auge de las redes sociales, el uso y los estudios sobre estas metodologías activas han proliferado ostensiblemente (Pérez-Pueyo \& Hortigüela, 2020). Los estudios realizados hasta la fecha se han centrado en las siguientes características: establecer cuáles son las principales metodologías activas utilizadas (Gómez, Chaparro, Felices \& Cózar, 2020; Nurash et al., 2020; Sánchez-Cortés \& Suárez, 2019), relacionar la utilización de las mismas y el rendimiento académico del alumnado (Gamazo, Martínez-Abad, Olmos-Miguelañez \& Rodríguez-Conde, 2018; Gil et al., 2018; Teig, Scherer \& Nilsen, 2018), explicitar diferencias en la utilización de los enfoques activos (Aparicio-Ting, Slater \& Kurz, 2019; Fernández-de-Álava \& Quesada-Pallarés, 2017; Fernández-Jiménez, Fernández \& Polo, 2017; Rodríguez-García \& Arias-Gago, 2019), implementar experimentalmente algunas de estas metodologías (Cantó, De Pro \& Solbes, 2016; García-Valcárcel \& Tejedor, 2018; Nieto \& Alfageme-González, 2017) y, finalmente, aparecen los que se centran en las concepciones, opiniones y percepciones que los docentes manifiestan hacia las mismas (Crisol, 2012; De Miguel, 2005; Gil-Flores, 2017; Jiménez-Hernández, González-Ortiz \& Tornel-Abellán, 2020).

El arraigo metodológico que se ha producido en la última década ha generado que el número de investigaciones para cuantificar el uso y las concepciones, percepciones y opiniones del profesorado hacia las metodologías activas haya aumentado significativamente (Hynes, 2017; Jiménez-Hernández et al., 2020). Este último aspecto también puede extrapolarse a los instrumentos creados para la medición de las variables mencionadas. A este respecto, los principales instrumentos revisados en la literatura vinculada al tema, ordenados cronológicamente, son los siguientes:

- University Student's Expectations of Teaching (USET) (Sander, Stevenson, King \& Coates, 2000): sobre las percepciones de estudiantes y profesores sobre métodos de enseñanza y evaluación docente.

- Instructions Preferred Questionnaire (IPQ) (Hativa \& Birenbaum, 2000): sobre las preferencias y expectativas del profesorado respecto a los métodos de enseñanza.

- Inventario de enfoques de enseñanza (ATI) (Trigwell \& Prosser, 2004): sobre el enfoque de enseñanza implementado por un docente en una situación determinada.

- Cuestionario de modalidades de enseñanza centradas en el desarrollo de competencias (De Miguel, 2005): sobre las modalidades, metodologías y sistemas de evaluación más adecuados para un aprendizaje basado en competencias.

- Documento sobre metodologías activas para la formación de competencias (Fernández, 2006): sobre el conocimiento y los prerrequisitos previos que necesita el profesorado para implementar metodologías activas.

- Encuestas para la renovación de las metodologías educativas en la Universidad (Ministerio de Educación y Formación Profesional) (MEFP, 2006): sobre la medición de dimensiones que facilitan o ponen barreras al uso de metodologías en el ámbito universitario (compromiso institucional, formación, dotación de recursos e incentivos, y procesos de evaluación y mejora).

- Cuestionario sobre las perspectivas de los estudiantes sobre el uso de las metodologías activas en el Grado de Maestro de Educación Infantil de la Universidad de Murcia (Vallejo \& Molina, 2011): sobre las percepciones que los estudiantes tienen sobre la utilización que realiza su profesorado de las metodologías activas.

- Cuestionario de opinión y percepción sobre el uso de metodologías activas del Profesorado de la Universidad de Granada (OPPUMAUGR) (León \& Crisol, 2011): sobre la opinión del profesorado y alumnado sobre aspectos limitantes y/o facilitadores del uso de metodologías activas (renovación metodológica, espacios y recursos, actividad docente, conocimiento y formación sobre metodologías activas, mejoras en la docencia, y lección magistral versus metodologías activas).

- Cuestionario para evaluar la organización, funcionamiento y gestión de los rincones en Educación Infantil (Conde, García \& Delgado-García, 2019): sobre la utilización de los rincones como enfoque metodológico por parte del profesorado de Educación Infantil.

- Cuestionario de opinión y percepción sobre el uso de metodologías activas en la Educación Obligatoria de la ciudad de León (España) (OPPUMAEOL) (Rodríguez-García \& Arias-Gago, 2019): sobre el uso de diversos enfoques metodológicos y la predisposición del profesorado hacia las metodologías activas.

- Instrumento para la medición de metodologías activas en la universidad (Jiménez-Hernández et al., 2020): sobre el conocimiento y uso de métodos activos por parte del profesorado universitario.

El denominador común de los instrumentos citados es que, en la mayoría de los casos, se focalizan en la Educación Superior o en una determinada etapa educativa. Por este motivo, es relevante continuar con el diseño, creación y validación de instrumentos que permitan medir las concepciones, opiniones y percepciones del profesorado de diversas etapas educativas hacia las metodologias activas ya que, las concepciones que los docentes tienen hacia las mismas se vinculan con su utilización en el aula (Hynes, 2017).

Ante la realidad expuesta, esta investigación tuvo como principal objetivo desarrollar y diseñar un instrumento ajustado a los criterios de fiabilidad y validez, y adecuado psicométricamente 
para valorar las percepciones, concepciones y opiniones que la población española de docentes no universitarios dispone hacia las metodologías activas. Para este propósito y considerando a López y Pantoja (2018), se utilizó un instrumento escalar como medio de medida apropiado y relevante al objetivo de la investigación.

\section{Método}

El presente trabajo tiene su origen en un proyecto de investigación de la Universidad de León que tiene por objeto establecer diferencias en la utilización de diferentes enfoques metodológicos activos entre el profesorado nacional no universitario. Por tanto, la investigación se dirige hacia la validación y análisis de las características psicométricas de la Escala Cuantificadora de las Opiniones y Concepciones Docentes hacia la Utilización de Metodologías Docentes Activas (escala OCDUMA), creada adhoc para el proceso de investigación sobre metodologías activas. Se parte de un diseño de investigación cuantitativo por encuesta para recopilar información de una amplia población y, de este modo, obtener estadísticas representativas de la misma.

\section{Participantes}

Se siguió un muestreo aleatorio por conglomerados, en el que la población se dividió en 19 estratos que se corresponden con las 17 comunidades autónomas del territorio español y las 2 ciudades autónomas de Ceuta y Melilla. Se consideraron como conglomerados los diferentes centros educativos de Educación Infantil, Primaria, Secundaria y Bachillerato. La muestra total estuvo compuesta por 12490 docentes de Educación Infantil, Primaria, Secundaria y Bachillerato $(n=12490)$, representativa de la población de docentes no universitarios del territorio español $(n=765098)$ (MEFP, 2020), con un margen de error del $2 \%$ y un nivel de confianza del $99 \%$.

A nivel sociodemográfico, la muestra resultante estuvo compuesta por 4580 hombres (36.67\%) y 7910 mujeres (63.33\%). En la tabla 1 puede observarse la distribución de la muestra por género entre las 17 comunidades autónomas y las 2 ciudades autónomas de Ceuta y Melilla.

Tabla 1.

Distribución de docentes por comunidad autónoma

\begin{tabular}{lccccccc}
\hline $\begin{array}{c}\text { Comunidad } \\
\text { autónoma }\end{array}$ & $\mathrm{n}$ & Hombres & Mujeres & $\begin{array}{c}\text { Comunidad } \\
\text { autónoma }\end{array}$ & $\mathrm{n}$ & Hombres & Mujeres \\
\hline Andalucía & 1620 & 765 & 855 & La Rioja & 360 & 150 & 210 \\
Aragón & 390 & 160 & 230 & Navarra & 855 & 240 & 615 \\
Asturias & 870 & 200 & 670 & Madrid & 530 & 160 & 370 \\
Cantabria & 710 & 265 & 445 & Murcia & 200 & 70 & 130 \\
Castilla La & 855 & 285 & 570 & País Vasco & 565 & 180 & 385 \\
Mancha & 1770 & 710 & 1060 & Islas Baleares & 335 & 65 & 270 \\
Castilla y León & & & & & & & \\
Comunidad & 505 & 235 & 270 & Islas Canarias & 555 & 215 & 335 \\
Valenciana & 810 & 240 & 570 & Ceuta & 100 & 45 & 55 \\
Cataluña & 435 & 220 & 215 & Melilla & 100 & 20 & 80 \\
Galicia & 925 & 355 & 570 & Total & 12490 & 4580 & 7910 \\
\hline Extremadura & & & & & & &
\end{tabular}

Nota. Fuente: elaboración propia.

\section{Instrumento}

La escala utilizada para llevar a cabo este estudio se ha denominado OCDUMA (Escala Cuantificadora de las Opiniones y Concepciones Docentes hacia la Utilización de Metodologías Docentes Activas). Fue creada ad-hoc para la investigación, considerando todos los instrumentos previos mencionados en la introducción. Tras esta primera revisión de la literatura, se definieron los ítems, siguiendo los criterios de relevancia, pertinencia y univocidad, establecidos por Alaminos y Castejón (2006).

La escala quedó conformada por 24 ítems cerrados, que debían ser valorados con una escala tipo Likert de 4 grados (1-totalmente en desacuerdo, 2-en desacuerdo, 3-de acuerdo, 4-totalmente de acuerdo). Posteriormente, las preguntas se dividieron en 4 dimensiones: mejoras en la docencia; reticencias hacia el uso; recursos, espacios y tiempos; y formación en metodologías activas. Antes de la escala, aparece una parte vinculada a variables sociodemográficas.

La escala fue validada por medio del procedimiento de juicio de expertos en el que participaron 9 doctores en Ciencias de la Educación con una experiencia igual o superior a 5 años. Estos expertos valoraron la relevancia, pertinencia y univocidad de los 24 ítems e hicieron observaciones cuando fueron necesarias. Estas valoraciones dieron como resultado la supresión de 3 ítems, por no ajustarse a 2 de los 3 criterios establecidos (relevancia, pertinencia y univocidad), por lo que la escala quedó compuesta por 21 ítems agrupados en 4 dimensiones.

Tras la administración de la escala y la posterior implementación del análisis factorial exploratorio esta quedó finalmente conformada por 18 ítems agrupados en 5 dimensiones (tabla 2):

- Dimensión 1. Mejoras en la docencia. Dirigida a la valoración de las mejoras que la utilización de metodologías activas trae consigo en los procesos de enseñanza-aprendizaje.

- Dimensión 2. Reticencias hacia el uso. Asociada a la valoración de aspectos internos de los docentes (miedo, envejecimiento y motivación) que frenan la utilización de metodologías activas.

- Dimensión 3. Espacios y Recursos. Focalizada en cuantificar cómo la infraestructura y recursos de los centros educativos facilitan o dificultan el uso de metodologías activas.

- Dimensión 4. Factores limitantes. Vinculada a valorar aspectos externos a los docentes (currículo, pruebas externas y burocracia) que limitan la utilización de metodologías activas.

- Dimensión 5. Formación en metodologías activas. Relacionada con valorar la idoneidad de la formación inicial y permanente sobre metodologías activas que se imparte al profesorado.

\section{Procedimiento de recogida y análisis de datos}

Establecidos los 19 estratos coincidentes con las comunidades y las 2 ciudades autónomas de Ceuta y Melilla, se administró el cuestionario a todos los centros educativos de la población de docentes no universitarios de cada comunidad autónoma (conglomerados). Para ello, se recopilaron los correos electrónicos institucionales de todos los centros educativos de la población, accediendo a los mismos por medio de la base de datos estatal de centros docentes no universitarios (MEFP, 2020).

Una vez recopilados los correos, la escala fue digitalizada utilizando el software Microsoft Forms para, seguidamente, ser enviada a los directores de los centros educativos junto con el consentimiento informado y una misiva explicativa del objetivo 
Tabla 2.

Dimensiones e items de la escala

\begin{tabular}{ll}
\hline \multicolumn{1}{c}{ Dimensiones } & \multicolumn{1}{c}{ Ítems } \\
\hline & $\begin{array}{l}\text { 1. El uso de metodologías activas potencia los } \\
\text { conocimientos, procesos y actitudes del alum- } \\
\text { nado. }\end{array}$ \\
& $\begin{array}{l}\text { 2. Las metodologías activas potencian las com- } \\
\text { petencias del alumnado (autonomía, uso de las } \\
\text { TIC, metacognición, emprendimiento, habilida- } \\
\text { des sociales...) }\end{array}$
\end{tabular}

3. Las metodologías activas mejoran y aumentan la interacción docentes-alumnos.

Factor 1. Mejoras

4. El uso de metodologías activas fomenta la en la docencia interdisciplinariedad de contenidos.

5. Las metodologías activas implican modelos de evaluación comprensivos con el alumnado.

6. La formación en metodologías activas es necesaria para que se genere el cambio metodológico.

7. El desarrollo de metodologías activas necesita de la coordinación del profesorado.

8. Las metodologías activas se centran en el aprendizaje del alumnado más que en el proceso de enseñanza del docente.

9. El envejecimiento docente dificulta la implementación de metodologías activas.

Factor 2. Reticen- 10. El miedo al cambio explica el escaso uso de cias hacia el uso metodologías activas.

11. La falta de motivación por la ausencia de incentivos frena el uso de metodologías activas.

12. Los espacios destinados a la docencia en los centros facilitan el uso de metodologías activas.

Factor 3. Espacios y recursos

13. Los centros cuentan con los recursos necesarios para implementar metodologías activas.

14. El currículo y los planes de estudio dificultan la implementación de metodologías activas.

Factor 4. Factores limitantes

15. Las pruebas externas dificultan la implementación de metodologías activas.

16. El uso de las metodologías activas implica un mayor trabajo burocrático y organizativo por parte de los docentes.

17. La formación inicial en metodologías activas

Factor 5. Formaaportadas desde la universidad es adecuada.

ción en métodos activos

18. La formación impartida por los centros de formación del profesorado es amplia y adecuada.

Nota. Fuente: elaboración propia

principal de la investigación y de las instrucciones a seguir, para así poder administrar la misma a todo el profesorado del centro educativo. Después, los directores de los centros que aceptaron participar reenviaron la escala al correo corporativo de los docentes de su centro, quienes la completaron de forma on-line, voluntaria y anónima, en un tiempo aproximado de 5 minutos.

Una vez finalizada la recogida de datos, se analizó el contenido de los ítems a través de un análisis factorial exploratorio aplicado bajo el método de extracción de componentes principales y rotación Varimax, previa comprobación de la adecuación de este análisis, utilizando la prueba KMO y Barlett. Para estos análisis se utilizó el software SPSS en su versión 26.

A continuación, se aplicó un análisis factorial confirmatorio, que permitió evaluar el ajuste del modelo por medio de un análisis de ecuaciones estructurales (SEM) con el Software AMOS SPPS en su versión 26, utilizando medidas de ajuste absoluto, incrementales y de parsimonia. Asimismo, se comprobó la consistencia interna de la escala con el coeficiente Alfa de Cronbach y el análisis de varianza promedio extraída (AVE). También se comprobó la fiabilidad del modelo por mediación de las correlaciones entre las dimensiones latentes de la escala, a través del coeficiente $\mathrm{R}$ de Pearson.

En último lugar, sobre la totalidad de la muestra, se llevó a cabo un estudio descriptivo-comparativo a través de la variable de agrupación género, utilizando la prueba t-student para establecer diferencias.

\section{Resultados}

\section{Análisis factorial exploratorio}

El análisis factorial exploratorio (AFE en adelante) permitió establecer una comparación entre la estructura teórica inicial del instrumento y su composición real, permitiendo mejorar la escala considerando los resultados obtenidos con base a su validez. Para este propósito, se tuvieron en cuenta los siguientes criterios para valorar la retención factorial: valores propios mayores que 1 , interpretación de factores de prueba y análisis y visualización del gráfico de sedimentación (Williams, Onsman \& Brown, 2010). Así pues, la prueba Kaiser-Meyer Olkin [KMO=.819], el test de esfericidad de Barlett $[p=.000]$ y la raíz del residuo cuadrático promedio $[\mathrm{RMSR}=.492]$, muestran resultados que se pueden considerar adecuados. Establecida la verificación criterial, se aplicó el AFE a la escala en su versión original (21 ítems y 4 dimensiones).

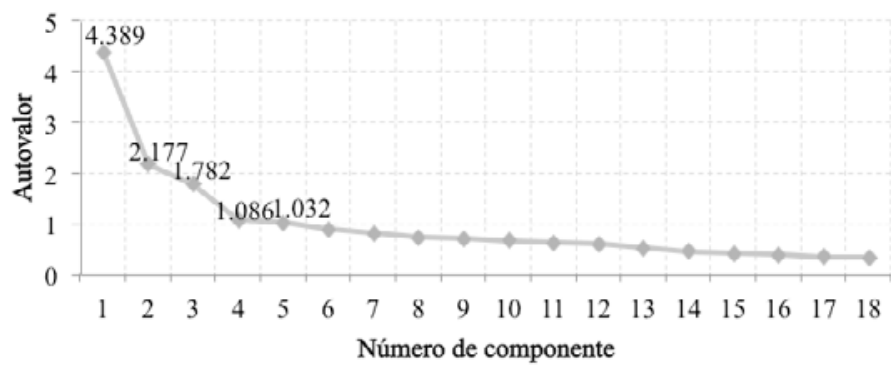

Figura 1. Gráfico de sedimentación

El AFE aplicado instó a establecer 5 factores, ya que los 5 componentes de los autovalores iniciales superan el valor 1 y explican el $54.705 \%$ de la varianza. Las comunalidades fueron $>.5$ en todos los ítems salvo en 3 (la carga docente dificulta la puesta en práctica de metodologías activas, la ratio actual de alumnos por grupo es adecuada para implementar metodologías activas y las TIC son necesarias para implementar metodologías activas). Este aspecto, junto con los valores $<.5$ en la matriz de componentes rotados y el aumento de la fiabilidad en el Alfa de Cronbach al suprimir los 3 elementos, instó a la supresión de los mismos (Barbero, Vila \& Holgado, 2011).

Cuando se ejecutó nuevamente el AFE sin los ítems indicados, se obtuvo un valor $\mathrm{KMO}=.897$ con alta significación en la prueba de esfericidad Barlett $\mathrm{p}=.000$, un $\mathrm{RMSR}=.398$ y comunalidades $>.5$ en todos los ítems del cuestionario. Los 5 factores extraídos explican esta vez el $58.89 \%$ de la varianza total y adquieren valores $>1$ en los autovalores iniciales de la varianza total explicada, tal y como puede observarse en la figura 1, correspondiente al gráfico de sedimentación.

En última instancia, la matriz de componentes rotados que puede observarse en la tabla 3, muestra el peso factorial de cada 
ítem agrupado en cada uno de los 5 factores, los cuales se corresponden con las 5 dimensiones de la tabla 2. Todas las cargas de los ítems son $>.5$ aspecto que indica adecuación factorial (Tabachnick \& Fidell, 2013).

Tabla 3.

Matriz de componentes rotados

\begin{tabular}{|c|c|c|c|c|c|}
\hline \multirow{2}{*}{ Ítem } & \multicolumn{5}{|c|}{ Factor } \\
\hline & 1 & 2 & 3 & 4 & 5 \\
\hline 1 & .810 & & & & \\
\hline 2 & .810 & & & & \\
\hline 3 & .787 & & & & \\
\hline 4 & .769 & & & & \\
\hline 5 & .743 & & & & \\
\hline 6 & 666 & & & & \\
\hline 7 & .606 & & & & \\
\hline 8 & .519 & & & & \\
\hline 9 & & .774 & & & \\
\hline 10 & & .703 & & & \\
\hline 11 & & .667 & & & \\
\hline 12 & & & .849 & & \\
\hline 13 & & & .848 & & \\
\hline 14 & & & & .766 & \\
\hline 15 & & & & .612 & \\
\hline 16 & & & & .570 & \\
\hline 17 & & & & & .799 \\
\hline 18 & & & & & .791 \\
\hline
\end{tabular}

Nota: nomenclatura de ítems en tabla 2

\section{Análisis factorial confirmatorio}

La confirmación del modelo obtenido por medio del AFE se ha realizado a través de un análisis factorial confirmatorio (AFC), mediante modelos de ecuaciones estructurales (SEM). El método de estimación implementado ha sido el de máxima verosimilitud y el modelo puede observarse en la figura 2 donde aparecen los índices de ajuste, regresión y correlación entre los factores.

La bondad de ajuste al modelo aparece reflejada en la tabla 4, considerando las medidas de ajuste absoluto, las medidas de incremento y las de parsimonia. En el seno de las medidas de ajuste absoluto al modelo: el estadístico Chi-Cuadrado $\left(X^{2}=5.82\right)$, para el que la recomendación de valores oscila entre 2 y 5 , es ligeramente superior, aunque se debe indicar que este índice es sensible cuando la muestra supera los 200 participantes, por lo que hay que tener en cuenta otros índices, como la raíz media cuadrática residual [RMSEA=.42], que obtuvo un valor de ajuste aceptable, al ubicarse los valores por debajo de .5 (Arias, 2008).

Las medidas incrementales (índice de ajuste comparativo$\mathrm{CFI}=.951$, índice de Tucker-Lewis-TLI=.935, índice de ajuste normalizado-NFI=.949, índice de ajuste incremental-IFI=.951) muestran valores cercanos o superiores a .95 (Arias, 2008), los cuales garantizan la validez del instrumento a nivel de constructo y confirman el modelo propuesto.

En último término, las medidas de parsimonia vinculadas al PRatio=.902 y al índice de ajuste normalizado de parsimonia, PNFI=.916, disponen de valores aceptables por encima de .9. Por su parte, el índice de bondad de ajuste de parsimonia, PGFI $=.581$, muestra valores aceptables ubicados en el rango $.5 \mathrm{y}$
.7 (Arias, 2008). Todos estos valores nos permiten determinar un ajuste idóneo del modelo que garantiza la validez de la escala.

La totalidad de los parámetros del modelo devuelven cargas factoriales con puntuaciones asociadas a cada factor por encima de .5 en todos los casos y, en promedio, por encima de .7 , lo que permite concluir que los diferentes ítems de la escala realizan una aportación aceptable a las dimensiones de las que proceden (Arias, 2008).

Tabla 4.

Índices de ajuste al modelo

\begin{tabular}{lcccccccccccc}
\hline Medidas & \multicolumn{4}{c}{ Ajuste absoluto } & \multicolumn{3}{c}{ Incrementales } & \multicolumn{2}{c}{ Parsimonia } \\
\hline Índices & $\mathrm{X}^{2}$ & df & $\mathrm{p}$ & RMSEA & CFI & TLI & NFI & IFI & PRATIO PGFI PNFI \\
Valores & 5.82 & 138 & .071 & .42 & .951 & .935 & .949 & .951 & .902 & .581 & .916 \\
\hline
\end{tabular}

Nota. Fuente: elaboración propia

\section{Análisis de fiabilidad y de correlación entre factores}

La fiabilidad y la consistencia interna de la versión final de la escala fue analizada utilizando el índice Alfa de Cronbach y la Varianza Promedio Extraída (AVE). En consecuencia, el coeficiente Alfa de Cronbach para la totalidad de la escala es de .871, valor considerado como bueno según George y Mallery (2001). La tabla 5 muestra los coeficientes de cada uno de los 5 factores que componen la escala, en todos ellos, los índices se encuentran por encima de .7, aspecto que dota al instrumento de una elevada consistencia interna (Merino-Soto, 2016).

Tabla 5.

Consistencia interna del instrumento

\begin{tabular}{lcc}
\hline Dimensión & $\begin{array}{c}\text { Alfa de Cron- } \\
\text { bach }\end{array}$ & AVE \\
\hline Factor 1: mejoras en la docencia & $\alpha=.954$ & .764 \\
Factor 2: reticencias hacia el uso & $\alpha=.818$ & .622 \\
Factor 3: espacios y recursos & $\alpha=.870$ & .644 \\
Factor 4: factores limitantes & $\alpha=.789$ & .568 \\
Factor 5: formación en métodos & $\alpha=.831$ & .634 \\
activos & $\alpha=.871$ & .623 \\
\hline Total &
\end{tabular}

Nota. Fuente: elaboración propia

Para concluir el análisis de fiabilidad, se realizó un análisis de varianza promedio extraída (AVE), el cual arrojó valores >.5 para los 5 factores del cuestionario aspecto que, considerando a Calderón, Arias-Estero, Meroño y Méndez-Giménez (2018), corrobora la adecuada fiabilidad de la escala.

El siguiente análisis consistió en establecer un estudio correlacional entre las 5 dimensiones de la escala ya corroboradas tras los análisis factoriales. Para este propósito, se implementó la prueba de correlación de Pearson, reflejada en la tabla 6. Los resultados indican que existen correlaciones significativas positivas entre todas las dimensiones de la escala. El coeficiente $\mathrm{R}$ es superior a .6, lo que según Pérez, García, Gil y Galán (2009), se asocia con una relación alta. Asimismo, la significación bilateral es $<.01$ en todos los ítems. 


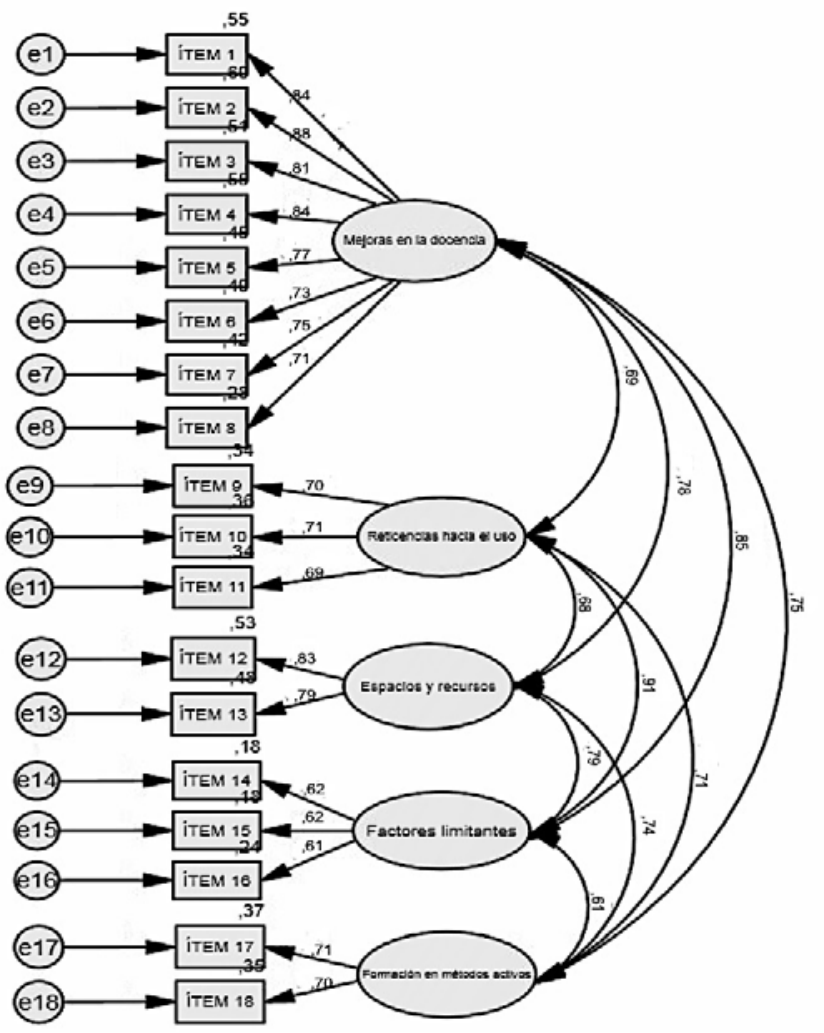

Figura 2. Modelo de 5 Factores (AFC)

Tabla 6

Correlaciones bivariadas para las 5 dimensiones latentes de la escala

\begin{tabular}{|c|c|c|c|c|c|c|}
\hline & & Factor 1 & Factor 2 & Factor 3 & Factor 4 & Factor 5 \\
\hline \multirow{3}{*}{ Factor 1} & $R$ & 1 & $.69^{* *}$ & $.78^{* *}$ & $.85^{* *}$ & $.75^{* *}$ \\
\hline & $p$ & & .000 & .000 & .000 & .000 \\
\hline & $n$ & 12490 & 12.490 & 12490 & 12.490 & 12490 \\
\hline \multirow{3}{*}{ Factor 2} & $R$ & & 1 & $.68^{* *}$ & $.91^{* *}$ & $.71^{* *}$ \\
\hline & $p$ & & & .000 & .000 & .000 \\
\hline & $n$ & & 12490 & 12.490 & 12.490 & 12.490 \\
\hline \multirow{3}{*}{ Factor 3} & $R$ & & & 1 & $.79^{* *}$ & $.74^{* *}$ \\
\hline & $p$ & & & & .000 & .000 \\
\hline & $n$ & & & 12490 & 12.490 & 12.490 \\
\hline \multirow{3}{*}{ Factor 4} & $R$ & & & & 1 & $.61^{* *}$ \\
\hline & $p$ & & & & & .000 \\
\hline & $n$ & & & & 12490 & 12.490 \\
\hline & $R$ & & & & & 1 \\
\hline
\end{tabular}

Factor $5 p$

12490

Nota. Fuente: elaboración propia

\section{Análisis descriptivo y comparativo}

La tabla 7 muestra los resultados descriptivos obtenidos por cada ítem en la escala final. El factor 1, mejoras en la docencia, es el que obtiene valores promedio más altos en la mayoría de ítems por los que se compone, estando los promedios siempre por encima de 3 [ $\bar{x}$ global del factor=3.42]. Esta circunstancia indica que existe acuerdo en el hecho de que las metodologías activas suponen mejoras en los procesos de enseñanza-aprendizaje. A nivel de género, en todos los 8 ítems obtienen puntuaciones promedio más elevadas las mujeres, con diferencias estadísticamente significativas en todos ellos $(\mathrm{p}<.01)$.

Tabla 7.

Estadísticos descriptivos y prueba t-student por género Factores Ítem Género $\overline{\mathrm{x}}$ Med. Dt. Min. Max. $\mathrm{n}$ t Sig. $\left(\mathrm{p}<.01^{* *}\right)$

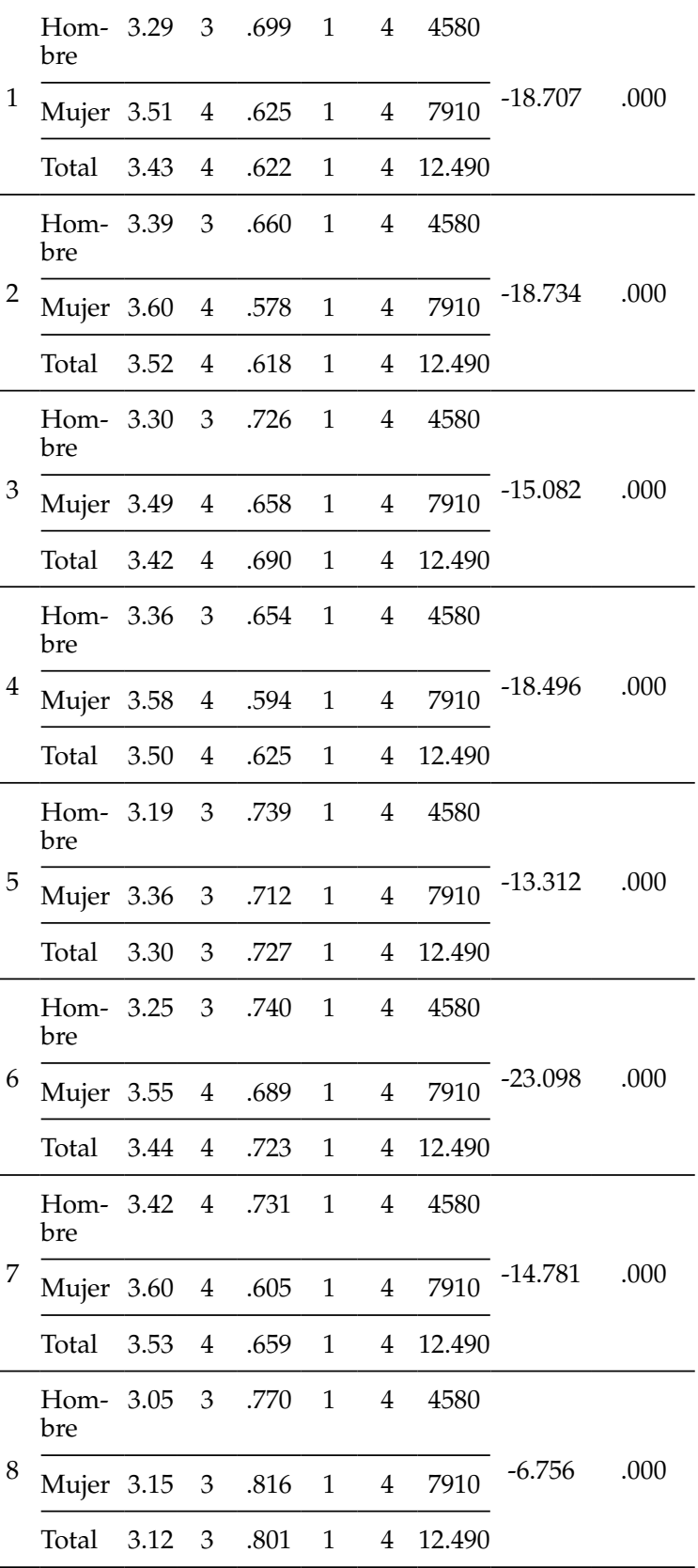




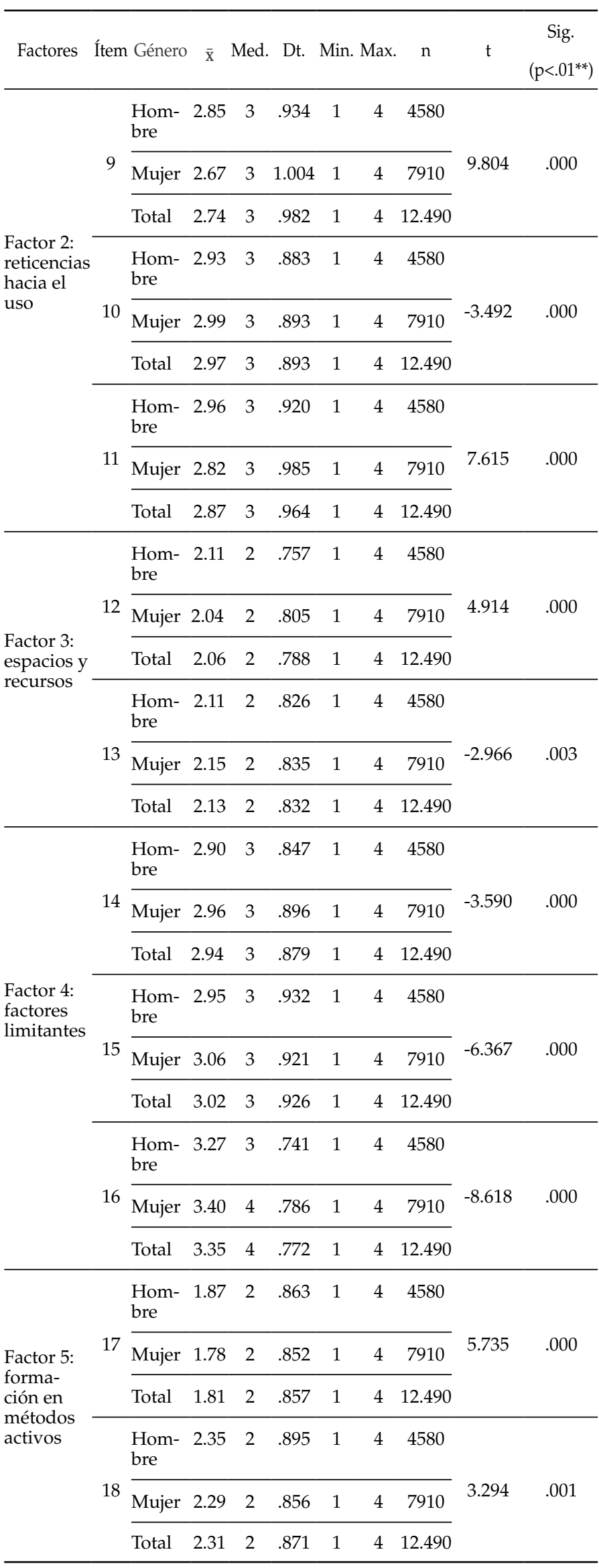

Nota. Fuente: elaboración propia
En el factor 2, reticencias hacia el uso, los valores promedio se sitúan en los 3 ítems por debajo de 3 [ $\overline{\mathbf{x}}$ global del factor=2.86]. El miedo al cambio es considerado el aspecto que más reticencia genera hacia la utilización de metodologías activas. En el presente caso, el género masculino es el que manifiesta mayores reticencias en 2 de los 3 ítems.

En el factor 3, espacios y recursos, se obtienen algunos de los valores promedio más bajos -cercanos a 2 [ $\overline{\mathbf{x}}$ global del factor $=2.095]-$, aspecto que se traduce en que los docentes perciben que los espacios y los recursos de los centros educativos no facilitan el uso de metodologías activas. En este factor, se producen también diferencias estadísticamente significativas por género entre sus 2 ítems, en un caso a favor de los hombres (ítem 12) y en otro de las mujeres (ítem 13) $(\mathrm{p}<.01)$.

En el factor 4 , factores limitantes, se obtienen los segundos valores promedio más altos, por encima de 3 [ $\bar{x}$ global del factor $4=3.10]$. Esto indica que existe consenso entre la muestra en el hecho de que factores limitantes externos impiden el uso de metodologías activas. A nivel de diferencias, las mujeres perciben una mayor limitación en los 3 ítems por los que se compone el factor.

En el factor 5, formación en métodos activos, la muestra obtiene los valores promedio más bajos, cercanos a 2 [ $\bar{x}$ global del factor $5=2.06]$, lo que indica concepciones bajas sobre la formación inicial y permanente del profesorado en metodologías activas. Además, se producen diferencias significativas a favor de los hombres en los 2 ítems asociados a este factor $(\mathrm{p}<.01)$.

\section{Discusión y conclusiones}

Desarrollar procesos de enseñanza-aprendizaje en los que el alumnado es el protagonista y se encarga de planificar, organizar y evaluar sus procesos de aprendizaje, actualmente no solo cobra un gran relevancia, sino que se convierte en un asunto primordial para la comunidad educativa como consecuencia del cambio de paradigma que se está produciendo. Por lo tanto, la implementación de metodologías activas en las aulas de las diferentes etapas es un medio esencial para lograr el cambio de paradigma citado (De Miguel, 2005; Nurash et al., 2020).

El objetivo de esta investigación ha sido diseñar y validar un instrumento que permita medir con eficacia las concepciones, percepciones y opiniones que los docentes no universitarios tienen hacia las metodologías activas. La escala OCDUMA, tras un exhaustivo análisis psicométrico, ha confirmado la existencia de 5 dimensiones, denominadas de la siguiente forma: mejoras en la docencia, reticencias hacia el uso, espacios y recursos, factores limitantes y formación en métodos activos. La realización del AFE y el AFC, dieron como resultado una escala conformada por 18 ítems, tras la supresión de 3 de los 21 iniciales.

Las 5 dimensiones que conforman la escala se sustentan y corroboran en las bases teóricas e instrumentos de diversos autores vinculados a la literatura (Fernández, 2006; Hativa \& Birenbaum, 2000; Jiménez-Hernández et al., 2020; León \& Crisol, 2011; MEFP, 2006; Rodríguez-García \& Arias-Gago, 2019; Sander et al., 2000; Trigwell \& Prosser, 2004).

La dimensión 1, denominada mejoras en la docencia, conformada por 8 ítems (tabla 2), pone de manifiesto, al igual que sucede en estudios similares (Crisol, 2012; León \& Crisol, 2011; Rodríguez-García \& Arias-Gago, 2019), que diversas mejoras en los procesos de enseñanza-aprendizaje se relacionan con la utilización de metodologías activas.

La segunda dimensión, reticencias hacia el uso, quedó conformada por 3 ítems y corroboró que, factores del foro interno de los docentes, como el miedo al cambio, el envejecimiento y la falta de motivación por la ausencia de incentivos, generan que 
las metodologías activas no se institucionalicen en la docencia (León \& Crisol, 2011; Rodríguez-García \& Arias-Gago, 2019; Sander et al., 2000).

En lo respectivo a la tercera dimensión, espacios y recursos, esta al final quedó compuesta por 2 ítems, que tienen el propósito de valorar si los espacios y recursos que disponen los centros educativos facilitan la implementación de metodologías activas, lo que coincide con los instrumentos de diversos autores (Conde et al., 2019; De Miguel, 2005; León \& Crisol, 2011).

La cuarta dimensión, factores limitantes, está compuesta por 3 ítems, vinculados a factores limitantes externos al docente (currículo, planes de estudio, pruebas externas y mayor trabajo burocrático y organizativo) que limitan la institucionalización del uso de métodos docentes activos. Aunque en la mayoría de la literatura consultada los investigadores integran estos factores externos junto con las reticencias hacia el uso (Crisol, 2012; Jiménez-Hernández et al., 2020; León \& Crisol, 2011), el AFE instó a establecer 2 dimensiones diferenciadas.

Finalmente, la quinta dimensión, formación en métodos activos, se compone de 2 ítems, que sirven para valorar si la formación inicial y permanente del profesorado relacionada con el uso de metodologías es adecuada y promueve la utilización de las mismas. Precisamente, la mayoría de la literatura nacional e internacional también toma en consideración estos aspectos formativos (Crisol, 2012; Fernández, 2006; Hativa \& Birenbaum, 2000; Jiménez-Hernández et al., 2020; León \& Crisol, 2011; Autores, 2019; Sander et al., 2000; Trigwell \& Prosser, 2004).

Por otra parte, los análisis psicométricos realizados sobre la escala manifiestan una aceptable y robusta configuración de las diferentes dimensiones. El índice KMO superior a .8 y el índice de significatividad Bartlett <.01, permitieron aplicar los análisis factoriales. Tanto en el análisis correlacional, como en el estudio diferencial efectuado por género, se producen resultados significativos entre todas las dimensiones e ítems. En referencia al estudio diferencial, es necesario mencionar que, en la mayoría de ítems, el grupo de mujeres obtiene puntuaciones promedio mayores, lo que coincide con los trabajos de Crisol (2012) o Fuad, Zubaidah, Mahanal y Suarsini (2017).

Además, los estadísticos obtenidos en los AFE, AFC y en el análisis de consistencia interna, han permitido otorgar validez a la escala OCDUMA, de gran utilidad y fácil aplicación para la comunidad educativa ya que, permite conocer las opiniones, concepciones y percepciones del profesorado no universitario hacia las metodologías activas, en aras de mejorar los procesos de enseñanza-aprendizaje. Hynes (2017) establece que estas concepciones son predictores de la utilización metodológica que los docentes hacen en sus procesos de enseñanza.

A modo de conclusión, el hecho de que la mayoría de instrumentos para la medición de las opiniones, concepciones y percepciones hacia las metodologías activas se dirijan hacia la Educación Superior, otorga un valor novedoso y añadido a esta escala, ya que se centra en las etapas docentes no universitarias, donde el número de instrumentos es reducido y, en la mayoría de los casos, centrados en una única etapa educativa. Además, y como evidencia de la utilidad y aplicabilidad de la escala, es necesario mencionar que, estudiantes del Grado en Educación Primaria de las Universidades de Murcia y Castilla-La Mancha, han mostrado interés en la utilización de la misma para actividades académicas -solicitud de la escala como modelo para la creación de sus instrumentos de investigación y/o para la administración en la población de sus investigaciones- vinculadas al trabajo de fin de grado.

En lo referente a las limitaciones asociadas al instrumento destacan las siguientes: el posible sesgo de deseabilidad social en la respuesta de la muestra, la aplicación de la escala en un solo momento temporal o haber efectuado solamente el estudio diferencial en la variable género. Las futuras líneas de investigación podrían dirigirse a paliar algunas de las limitaciones expuestas, aplicando la escala depurada de nuevo a la misma muestra o efectuando estudios diferenciales de otras variables sociodemográficas (etapa educativa, experiencia docente, tipología de centro y comunidades autónomas), para determinar cómo influyen en las concepciones, opiniones y percepciones del profesorado hacia las metodologías activas.

\section{Referencias}

Alaminos, A., \& Castejón, J. L. (2006). Elaboración, análisis e interpretación de encuestas, cuestionarios y escalas de opinión. Alcoy: Marfil.

Aparicio-Ting, F. E., Slater, D. M., \& Kurz, E. U. (2019). Inquiry-Based Learning (IBL) as a Driver of Curriculum: A Staged Approach. Papers on Postsecondary Learning and Teaching, 3(1), 4451. https://bit.ly/2UnLF42

Arias, B. (2008). Desarrollo de un ejemplo de análisis factorial confirmatorio con LISREL, AMOS y SAS. En M. A. Verdugo, M. Crespo, M. Badía y B. Arias (Eds.), Metodología en la investigación sobre discapacidad. Introducción al uso de ecuaciones estructurales (pp. 75124). Salamanca: Publicaciones del INICO. https://bit.ly/3aql8eD

Barbero, M. I., Vila, E., \& Holgado, F. P. (2011). Introducción básica al análisis factorial. Madrid: UNED.

Calderón, A., Arias-Estero, J.L., Meroño, L., \& Méndez-Giménez, A. (2018). Diseño y Validación del Cuestionario de Percepción del Profesorado de Educación Primaria sobre la Inclusión de las Competencias Básicas. Estudios sobre Educación, 34, 67-97. https:/ / doi.org/10.15581/004.34.67-97

Cantó, J., De Pro, A., \& Solbes, J. (2016). ¿Qué ciencias se enseñan y cómo se hace en las aulas de Educación Infantil? La visión de los maestros en formación inicial. Enseñanza de las Ciencias, 34(3), 25-50. http:/ /dx.doi.org/10.5565/rev/ensciencias.1870

Conde, S., García, F. J., \& Delgado-García, M. (2019). Diseño y validación de un instrumento para analizar el trabajo por rincones en Educación Infantil. Estudios sobre Educación, 36, 53-83. https:/ / doi.org/10.15581/004.36.53-83

Crisol, E. (2012). Opinión y percepción del profesorado y de los estudiantes sobre el uso de metodologías activas en la universidad de Granada [Tesis Doctoral. Universidad de Granada]. Granada: Repositorio Universidad de Granada https://bit.ly/2RMfCZz

De Miguel, M. (2005). Modalidades de enseñanza centradas en el desarrollo de competencias. Orientaciones para promover el cambio metodológico en el marco del EEES. Oviedo: Servicio de publicaciones de la Universidad de Oviedo.

Fernández, A. (2006). Metodologías activas para la formación de competencias. Educatio siglo XXI, 24, 35-56. http://bit. ly/2WR4L2N

Fernández-de-Álava, M., \& Quesada-Pallarés, C. (2017). Estrategias de aprendizaje del profesorado Europeo: género y etapa educativa. Bordón, 69(1), 67-81. https://doi.org/10.13042/ Bordon.2016.38597

Fernández-Jiménez, C., Fernández, M., \& Polo, Mª T. (2017). Aplicación de la autoevaluación en una experiencia de aprendizaje basado en problemas con alumnado de educación en asignaturas relacionadas con la discapacidad. Estudios sobre Educación, 32, 73-93. https:/ / doi.org/10.15581/004.32.73-93

Fuad, N. M., Zubaidah, S., Mahanal, S., \& Suarsini, E. (2017). Improving Junior High Schools' Critical Thinking Skills Based on Test Three Different Models of Learning. International Journal of Instruction, 10(1), 101-116. https:/ / bit.ly/3fTr5B1 
Gamazo, A., Martínez-Abad, F., Olmos-Miguelañez S., \& Rodríguez-Conde M. J. (2018). Evaluación de los factores relacionados con la eficacia escolar en PISA. Un análisis multinivel. Revista de Educación, 379, 56-84. https://doi.org/10.4438/1988-592X-RE-2017-379-369

García-Valcárcel, A., \& Tejedor, F. J. (2018). Valoración del trabajo colaborativo en los procesos de enseñanza-aprendizaje en entornos escolares con alto nivel TIC. Estudios sobre Educación, 34, 155-175. https:/ / doi.org/10.15581/004.34.155-175

George, D., \& Mallery, M. P. (2001). SPSS for Windows step by step: A simple guide and reference. Boston: Allyn \& Bacon.

Gil, M., Cordero, J. M., \& Cristóbal, V. (2018). Las estrategias docentes y los resultados en PISA 2015. Revista de Educación, 379, 32-55. https://doi.org/10.4438/1988-592XRE-2017-379-368

Gil-Flores, J. (2017). Rasgos del profesorado asociados al uso de diferentes estrategias metodológicas en las clases de ciencias. Enseñanza de las Ciencias, 35(1), 175-192. https://doi. org/10.5565/rev/ensciencias. 1970

Gómez, C. J., Chaparro, A., Felices, M. M., \& Cózar, R. (2020). Estrategias metodológicas y uso de recursos digitales para la enseñanza de la historia. Análisis de recuerdos y opiniones del profesorado en formación inicial. Aula Abierta, 49(1), 65-74. https:/ / doi.org/10.17811/rifie.49.1.2020.65-74

Hativa, N., \& Birenbaum, M. (2000). Who prefers what? Disciplinary differences in student's preferred approaches to teaching and learning styles. Research in Higher Education, 41(2), 209235. https: / / doi.org/10.1023/A:1007095205308

Hynes, M. (2017). Students-as-producers: Developing valuable student-centered research and learning opportunities. International Journal of Research Studies in Education, 7(4), 1-13. https: / / doi.org/10.5861/ijrse.2017.1858

Jiménez-Hernández, D., González-Ortiz, J. J., \& Tornel-Abellán, M. (2020). Metodologías activas en la universidad y su relación con los enfoques de enseñanza. Profesorado. Revista de Currículum y Formación de Profesorado, 24(1), 76-94. https:/ /doi. org/10.30827/profesorado.v24i1.8173

Labrador-Piquer, M. J., \& Andreu-Andrés, M. A. (2008). Metodologías Activas. Grupo de innovación en metodologías activas. Valencia: Servicio de publicaciones de la Universidad Politécnica de Valencia. https:/ / bit.ly/2WbHcC8

León, M. J., \& Crisol, E. (2011). Diseño de cuestionarios (OPPOMAUGR Y OPEUMAUGR): La opinión y percepción del profesorado y de los estudiantes sobre el uso de metodologías activas en la universidad. Profesorado. Revista de currículum y formación del profesorado, 15(2), 272-298. https:/ / bit.ly/2Qd1Y06

López, M., \& Pantoja, A. (2018). Escala para valorar la percepción y grado de satisfacción del profesorado de Educación Primaria de la Comunidad Autónoma de Andalucía respecto a los procesos tutoriales. Revista Complutense de Educación, 29(1), 287-306. https: / / doi.org/10.5209/RCED.52286

MEFP (2006). Propuestas para la Renovación de las Metodologías Educativas en la Universidad. Madrid: Secretaría General Técnica del MEFP. http:/ / bit.ly/3e5YqH9
MEFP (2020, septiembre 25). Educabase. https://bit. ly/2GbWelN

Merino-Soto, C. (2016). Diferencias entre coeficientes alfa de Cronbach, con muestras y partes pequeñas: Un programa VB. Anales de Psicología, 32(2), 587-588. http://dx.doi.org/10.6018/ analesps.32.2.203841

Nieto, J. M., \& Alfageme-González, M. B. (2017). Enfoques, metodologías y actividades de formación docente. Profesorado. Revista de currículum y formación del profesorado, 21(3), 63-81. http:/ / bit.ly/3nYbFz1

Nurash, P., Kasevayuth, K., \& Intarakamhang, U. (2020). Learning programmes and teaching techniques to enhance oral health literacy or patient-centred communication for healthcare providers: A systematic review. European Journal of Dental Education, 24(1), 134-144. https:/ / doi.org/10.1111/eje.12477

OCDE (2019). PISA 2018. Technical report. París: OCDE Publishing. https://bit.ly/2HPszws

Oliver, M., McConney, A., \& Woods-McConney, A. (2019). The efficacy of inquiry based instruction in Science: a comparative analysis of six countries using PISA 2015. Research in Science education. https: / doi.org/10.1007/s11165-019-09901-0

Pérez, R., García, J. L., Gil, J. A., \& Galán, A. (2009). Estadística aplicada a la Educación. Madrid: Pearson Prentice Hall.

Pérez-Pueyo, A., \& Hortigüela, D. (2020). ¿Y si toda la innovación educativa no es positiva en Educación Física? Reflexiones y consideraciones prácticas. Retos, 37, 579-587. https://doi. org/10.47197/retos.v37i37.74176

Rodríguez-García, A., \& Arias-Gago, A.R. (2019). Uso de metodologías activas. Un estudio comparativo entre profesores y maestros. Brazilian Journal of Development, 5(6), 5098-5111. http:/ / bit.ly/37CvDKs

Sánchez-Cortés, I., \& Suárez, J. M. (2019). Métodos de enseñanza, compromiso y metas del profesorado en modalidad b-learning. Aula Abierta, 48(3), 311-320. https://doi.org/10.17811/ rifie.48.3.2019.311-320

Sander, P., Stevenson, K., King, M., \& Coates, D. (2000). University Student's Expectations of Teaching. Studies in Higher Education, 25(3), 309-323. https: / / doi.org/10.1080/03075070050193433

Tabachnick, B. G., \& Fidell, L. (2013). Using multivariate statistics. Boston: Pearson Education.

Teig, N., Scherer, R., \& Nilsen, T. (2018). More isn't always better: the curvilinear relationship between inquiry based teaching and student achievement in science. Learning and Instruction, 56, 20-29. https:/ / doi.org/10.1016/j.learninstruc.2018.02.006

Trigwell, K., \& Prosser, M. (2004). Development and Use of the Approaches to Teaching Inventory. Educational Psychology Review, 16(4), 409-424. https:/ / doi.org/10.1007/s10648-004-0007-9

Vallejo, M., \& Molina, J. (2011). Análisis de las metodologías activas en el grado de maestro en educación infantil: la perspectiva del alumnado. REIFOP, 14(1), 207-217. https: / / bit.ly/35Jvq5n

Williams, B., Onsman, A., \& Brown, T. (2010). Exploratory factor analysis: A five-step guide for novices. Journal of Emergency Primary Health Care (JEPHC), 8(3), Article 990399. https:/ /doi. org/10.33151/ajp.8.3.93 
